\title{
Cosmic mass functions from Gaussian stochastic diffusion processes
}

\author{
P. Schuecker, H. Böhringer, K. Arzner, and T. H. Reiprich \\ Max-Planck-Institut für extraterrestrische Physik, 85741 Garching, Germany \\ Received 4 July 2000 / Accepted 16 February 2001

\begin{abstract}
Gaussian stochastic diffusion processes are used to derive cosmic mass functions. To get analytic relations previous studies exploited the sharp $k$-space filter assumption yielding zero drift terms in the corresponding Fokker-Planck (Kolmogorov's forward) equation and thus simplifying analytic treatments significantly (excursion set formalism). In the present paper methods are described to derive for given diffusion processes and Gaussian random fields the corresponding mass and filter functions by solving the Kolmogorov's forward and backward equations including nonzero drift terms. This formalism can also be used in cases with non-sharp $k$-space filters
\end{abstract} \\ and for diffusion processes exhibiting correlations between different mass scales.
}

Key words. clusters: general - clusters - cosmology: theory

\section{Introduction}

The Press-Schechter (1974) formula of the cosmic mass function has found many important applications to basic problems in modern cosmology (e.g., Narayan \& White 1988; Cole \& Kaiser 1988, 1989; Henry \& Arnaud 1991; White \& Frenk 1991; White et al. 1993; Eke et al. 1996; Mo et al. 1996; Mo \& White 1996; Matarrese et al. 1997; Mathiesen \& Evrard 1998; Borgani et al. 1999). Although the approach appears questionable when the theoretical masses are compared with the group finder masses in detailed $N$-body simulations (e.g., Bond et al. 1991; White 1996) the overall statistical agreement is found to be sufficiently good (Efstathiou et al. 1988; Lacey \& Cole 1994, but see also Sect. 8). Improved versions relax the various assumptions of the original derivation (Lucchin \& Matarrese 1988; Bond et al. 1991; Lilje 1992; Cavaliere \& Menci 1994; Jedamzik 1995; Monaco 1995; Kitayama \& Suto 1996; Yano et al. 1996; Valageas \& Schaeffer 1997; Chiu et al. 1998; Lee \& Shandarin 1998).

Apart from providing a convenient fitting equation, the excursion set formalism in the form presented in Bond et al. (1991) serves as the basis for several fundamental theoretical developments aiming for a full treatment of structure formation in a hierarchically clustering universe. Extended theories for hierarchical clustering (Bower 1991), biasing schemes (Mo \& White 1996), and merging histories (Lacey \& Cole 1993; Kauffmann \& White 1993) are developed or can also be derived in the end by assum-

Send offprint requests to: P. Schuecker,

e-mail: peters@mpe.mpg.de ing the validity of the excursion set arguments introduced by Bond et al. to derive the Press-Schechter formula with the correct normalization and several related conditional probability densities.

For the derivation of the Press-Schechter function the excursion set formalism assumes that at a fixed spatial location the change of the density contrast successively smoothed with a decreasing filter scale, $R$, can be described as a sample path of an abstract diffusion process (Bond et al. 1991, see also Peacock \& Heavens 1990). The counting of subclumps (clouds-in-clouds) is avoided by computing the rates at which the sample paths meet at their largest mass extent a scale-independent critical density contrast barrier, $f_{\mathrm{c}}$. For this process Bond et al. derived a general Fokker-Planck (Kolmogorov's forward) equation consisting of a drift and of a diffusion term. The application of the sharp $k$-space filter condition yields a zero drift term so that the resulting much simpler FokkerPlanck equation can be solved easily and leads to the Press-Schechter mass function with the correct normalization.

Unfortunately, analytic results are obtained with the excursion set formalism only within the theoretical framework of Markovian processes (see below) and for the sharp $k$-space filter with a mass assignment scheme which clearly contradicts numerical experiments on the halo-by-halo basis. It is the basic aim of the present investigation to extend the excursion set formalism to non-sharp $k$-space filters but without leaving the framework of Markovian random processes and thus preserving the simplicity of the original approach. In the present context, Markov 
processes are assumed to be driven by deterministic and stochastic "forces", and have the simplifying property that when the process states, in form of amplitudes of filtered density contrasts, are known on a given mass scale and larger, the process states on smaller scales depend only on the actual scale and are independent of the larger scales it seems suprising that under this Markov condition, e.g., useful biasing schemes can be derived, but see below.

The present paper discusses both the Kolmogorov's forward and backward equation including nonvanishing drift terms. Analytic solutions are presented for several interesting cases offering the possibility to derive cosmic mass functions independent of the sharp $k$-space filter assumption. It will be shown that certain Gaussian stochastic diffusion processes lead to more realistic filter profiles and to correlations between different mass resolution scales which are expected to have important implications on various progenitor statistics and biasing schemes.

Section 2 gives an informal presentation of the basic concepts of the excursion set formalism. Section 3 summarizes the formal properties of the relevant diffusion processes and their sample paths. The connection between diffusion processes and mass functions is outlined in Sect. 4 . The relation between the basic pseudo time (resolution) variable and the filter radius is discussed in Sect. 5. In Sects. 6 and 7 the methods are applied to order-0 (Wiener) diffusion processes, characterized by drift coefficients which are independent of mass and filtered density contrast, and to order-1 (Ornstein-Uhlenbeck) diffusion processes, characterized by drift coefficients which are also independent of mass but which scale linearly with filtered density contrast. For both processes the corresponding mass and filter functions are derived. The results are summarized and discussed in Sect. 8. Appendix A gives an overview of the basic properties of order-1 diffusion processes.

Forthcoming papers will analyse the new mass assignment scheme, and will compare the mass functions presented here and others deduced from more complicated stochastic diffusion processes with detailed $N$-body simulations and with observed mass and luminosity functions of rich clusters of galaxies obtained with, e.g., the REFLEX cluster sample (Böhringer et al. 1998; Guzzo et al. 1999), and the HIFLUGCS cluster sample (T. H. Reiprich \& H. Böhringer, in preparation).

\section{Excursion set formalism}

The differential mass function, $n(M)$, gives the number of virialized objects of mass $M$, per unit mass and (comoving) volume element. We want to derive $n(M)$ using the field of density contrasts, $f(\boldsymbol{r})$, at the comoving coordinates, $\boldsymbol{r}$. Here, $f$ is defined by the ratio $f(\boldsymbol{r})=\rho(\boldsymbol{r}) / \bar{\rho}-1$, where $\rho(\boldsymbol{r})$ is the comoving mass density at $\boldsymbol{r}$, and $\bar{\rho}$ the mean comoving mass density. The following notation is simplified by neglecting any evolution of $f$ with cosmic time. However the final equations can be transformed to any epoch by multiplication with linear growth factors.
For the derivation of $n(M)$ it is assumed that the location where a virialized object of mass $M$ will be formed corresponds to the location where $f(\boldsymbol{r})$, filtered with the radius $R(M)$ to give $\tilde{f}(\boldsymbol{r}, R)$, has reached a universal critical threshold value, $f_{\mathrm{c}}$. The density field is divided into infinitesimal small volume elements. A sequence of filtered three-dimensional density fields is constructed with gradually decreasing filter radii. The excursion set formalism identifies the cumulative mass fraction $\propto n(>M)$ with the fraction of volume elements in which the density contrast lies above $f_{\mathrm{c}}$ when smoothed with any filter of radius greater than or equal $R(M)$.

At a fixed spatial location, $\boldsymbol{r}$, the sequence of filtered density contrasts, $\{\tilde{f}(\boldsymbol{r}, R) \mid R=\infty \ldots 0\}$, can be regarded as a one-dimensional trajectory. The statistical description of an ensemble of such sample paths gets especially simple when they result from a Gaussian random process with independent increments, i.e., from a Wiener process. For filtered density contrasts this is the case when $f(\boldsymbol{r})$ is a Gaussian random field uniquely described by the fluctuation power spectrum, $P(k)$, as a function of comoving wavenumber $k$, and when the top-hat filter in Fourier space, i.e., the sharp $k$-space filter (see Eq. (2) below) is used:

The power spectrum of the density field is defined via the Fourier transform of the unfiltered density contrasts, $f(\boldsymbol{k})=\mathrm{FT}[f(\boldsymbol{r})]$, by the expectation value $\mathrm{E}\left[f(\boldsymbol{k}) f^{*}\left(\boldsymbol{k}^{\prime}\right)\right]=(2 \pi)^{3} P(k) \delta_{\mathrm{D}}\left(\boldsymbol{k}-\boldsymbol{k}^{\prime}\right)$, where $\delta_{\mathrm{D}}$ is the Dirac delta distribution and ${ }^{*}$ assigns the complex conjugate. In this case the discrete change in $\tilde{f}(\boldsymbol{r}, R)$ caused by the transition $k_{R} \rightarrow k_{R}+\Delta k_{R}$ of the maximum comoving wavenumber, $k_{R}$, which passes the filter is a Gaussian random variable (each change of $\tilde{f}(\boldsymbol{r}, R)$ when $R$ is changed comes from the inclusion/exclusion of a new independent $k$ shell) with the variance $\Delta \sigma^{2}=\sigma^{2}\left[1 /\left(k_{R}+\Delta k_{R}\right)\right]-$ $\sigma^{2}\left[1 / k_{R}\right]$, where

$\sigma^{2}(R)=\frac{1}{(2 \pi)^{3}} \int_{0}^{\infty} \mathrm{d}^{3} k\left|W_{R}(k)\right|^{2} P(k)$.

The sharp $k$-space filter,

$W_{R}(k)=\left\{\begin{array}{l}1: k \leq k_{R}=1 / R \\ 0: \text { else }\end{array}\right.$

and Eq. (1) define (for spherically symmetric filters) a general resolution variable,

$\Lambda(R)=\frac{1}{2 \pi^{2}} \int_{0}^{\frac{1}{R}} \mathrm{~d} k k^{2} P(k)$.

For this choice, the $\tilde{f}(\Lambda)$ values perform some kind of Brownian motion with $\Lambda(R)$ as a pseudo time variable. Notice that for hierarchical clustering, $\Lambda$ as well as $\sigma^{2}$ increase with decreasing mass and filter scale. For $\Delta k_{R} \rightarrow 0$ a trajectory corresponds to one realization of a continuous limit of a simple random walk: the Wiener process.

Hence, the behaviour of these trajectories are governed by a simplified Fokker-Planck diffusion equation which gives the probability density, $\Pi$, to find trajectories with 
$\tilde{f}(\boldsymbol{r}, R)$ at $\Lambda$. In this picture the differential mass function can be obtained from the rate at which the random trajectories meet for the first "time" the absorbing barrier $f_{\mathrm{c}}$ at a given mass or $\Lambda[R(M)]$ scale. The final problem is to get solutions of the Fokker-Planck equation under the boundary conditions that all sample paths start at $\Lambda=0$ with $\tilde{f}=0$ and that absorption of a sample path at $\Lambda$ corresponds to $\Pi\left(\tilde{f}=f_{\mathrm{c}}, \Lambda\right)=0$ (see Sect. 6 for a more detailed discussion of these boundary conditions).

The excursion set formalism in the form presented above provides a simple mean to deduce the PressSchechter mass function. The major reason for the simplicity of the prescription results from the application of the sharp $k$-space filter. The choice of this filter appears to be rather ad hoc and not thoroughly motivated. Its strongly oscillating shape in configuration space does not seem to be optimal to frame the region of primordial material that ultimately collapses to form a virialized halo. Moreover, the absence of any covariances between different mass scales is not expected for realistic physical systems. In the following a more general (though more formal) description of the evolution of $\Pi$ is given, by considering a general diffusion process which offers the possibility to study also non-sharp filter functions and the resulting mass functions. It will be seen that certain diffusion processes lead to nonzero covariances between different mass scales without leaving the general framework of Markovian random processes. These processes are thus expected to yield more realistic formation histories and biasing schemes.

\section{Gaussian $\Lambda-\tilde{f}$ diffusion processes}

A Gaussian random field of the mass density contrast, $f$, is filtered on a continuous set of decreasing scales $R$, giving at a fixed spatial location in configuration space a continuous sequence of filtered density contrasts, $\tilde{f}$, at the scale $R$, providing a measure of the spatial resolution, $\Lambda(R)$. For scale-invariant power spectra, $P(k) \propto k^{n}$, the resolution variable has the form $\Lambda \propto R^{-(n+3)}$ (Eq. (3)). The diffusion process is restricted to a continuous $-\Lambda$, continuous - $\tilde{f}$ state space, where $\Lambda$ and $\tilde{f}$ assume the rôles of the usual time and spatial coordinates, respectively. In general it is expected that the sample paths of the diffusion process start with $\tilde{f}=\tilde{f}_{0}=0$ at $\Lambda=0$. Formally, a Gaussian $\Lambda-\tilde{f}$ diffusion process corresponds to a Markov process for which the transition probabilities, $\Pi\left(\tilde{f}^{\prime}, \Lambda+\Delta \Lambda, \tilde{f}, \Lambda\right)$, with the sharp value $\tilde{f}$ at $\Lambda$ and the random variable $\tilde{f}^{\prime}$ at $\Lambda+\Delta \Lambda$, satisfy a continuity condition (similar to Eq. (a) in Arnold 1974, p. 40), and

$$
\begin{array}{r}
\lim _{\Delta \Lambda \rightarrow 0} \frac{1}{\Delta \Lambda} \int_{\left|\tilde{f}^{\prime}-\tilde{f}\right| \leq \epsilon} \mathrm{d} \tilde{f}^{\prime}\left(\tilde{f}-\tilde{f}^{\prime}\right)^{m} \\
\Pi\left(\tilde{f}^{\prime}, \Lambda+\Delta \Lambda, \tilde{f}, \Lambda\right) \\
=A_{m}(\tilde{f}, \Lambda)+\mathrm{O}(\epsilon)
\end{array}
$$

exists for $\epsilon>0, m=1,2$, and $A_{m}=0$ for $m \geq 3$. The $A_{1}$ coefficient describes the deterministic drift or the instantaneous rate of change of the mean, and $A_{2}$ the stochastic diffusion or the instantaneous rate of change of the squared fluctuations of the sample paths giving the expectation, $E\left[\tilde{f}(\Lambda)-\tilde{f}\left(\Lambda^{\prime}\right)\right]^{m}=A_{m}(\tilde{f}, \Lambda) \Delta \Lambda+O(\Delta \Lambda)$. Note that the Markovian independence property introduces the simplifications mentioned in White (1997) concerning the application of the extended Press-Schechter theory to formation histories and biasing schemes: Because the Markov property is used to derive extended Press-Schechter, a say $10^{12} M_{\odot}$ halo at redshift $z=1$ does not "know" whether it will be incorporated into a rich cluster or remain in a void at $z=0$. The galaxy populations in protocluster and protovoid halos will thus be the same. However, the present investigation concentrates on a wider class of diffusion processes than studied by the extended PressSchechter approach, some of them exhibiting nonzero correlations between different mass scales. The expectation $E\left[\tilde{f}(\Lambda)-\tilde{f}\left(\Lambda^{\prime}\right)\right]^{m}$ leads with the inclusion of some additional heuristic arguments (see the formal treatments in, e.g., Friedman 1975; Kloeden \& Platen 1995) to the Itô stochastic differential equation,

$\mathrm{d} \tilde{f}(\Lambda)=-A_{1}(\tilde{f}, \Lambda) \mathrm{d} \Lambda+\sqrt{A_{2}(\tilde{f}, \Lambda)} \mathrm{d} w(\Lambda)$.

The second term on the right-hand side is the fundamental or Wiener stochastic process, i.e., a Gaussian process with the independent infinitesimal increments, $\mathrm{d} w(\Lambda)=$ $w(\Lambda+\mathrm{d} \Lambda)-w(\Lambda)=K(\Lambda) \mathrm{d} \Lambda$. A prescription for the construction of $w(\Lambda)$ is given by Eqs. (9) and (10). The stochastic "forces", $K(\Lambda)$, are Gaussian with infinite variance and no correlations between different $\Lambda$ shells. They drive the $\tilde{f}-\Lambda$ diffusion as illustrated by the Langevin-type equation, $\mathrm{d} \tilde{f} / \mathrm{d} \Lambda=-A_{1}(\tilde{f}, \Lambda)+\sqrt{A_{2}(\tilde{f}, \Lambda)} K(\Lambda)$. The increments, $\mathrm{d} \tilde{f}$, are of the order of the standard deviations of the fundamental process, that is, $\mathrm{d} \tilde{f} / \mathrm{d} \Lambda \approx \sqrt{\Delta \Lambda} / \Delta \Lambda$, which tends to infinity as $\Delta \Lambda \rightarrow 0$ (see also Wiener 1930; Doob 1942). The Itô formalism is used to handle the resulting almost surely continuous but almost surely nowhere differentiable sample paths in a mathematically well-defined way. An alternative would be the application of the Stratonovich formalism, but the results derived here do not depend on the specific choice.

Closely related to the stochastic diffusion Eq. (5) are Kolmogorov's forward (Fokker-Planck) equation,

$$
\begin{aligned}
\frac{\partial \Pi\left(\tilde{f}_{0}, \Lambda_{0}, \tilde{f}, \Lambda\right)}{\partial \Lambda} & =\mathcal{L}(\tilde{f}, \Lambda) \Pi\left(\tilde{f}_{0}, \Lambda_{0}, \tilde{f}, \Lambda\right), \\
\mathcal{L}(\tilde{f}, \Lambda) & =\frac{\partial}{\partial \tilde{f}} A_{1}(\tilde{f}, \Lambda)+\frac{1}{2} \frac{\partial^{2}}{\partial \tilde{f}^{2}} A_{2}(\tilde{f}, \Lambda),
\end{aligned}
$$

with the forward variables, $\tilde{f}$ and $\Lambda$, and Kolmogorov's backward equation,

$$
\begin{aligned}
\frac{\partial \Pi\left(\tilde{f}_{0}, \Lambda_{0}, \tilde{f}, \Lambda\right)}{\partial \Lambda_{0}} & =-\mathcal{L}^{\dagger}\left(\tilde{f}_{0}, \Lambda_{0}\right) \Pi\left(\tilde{f}_{0}, \Lambda_{0}, \tilde{f}, \Lambda\right), \\
\mathcal{L}^{\dagger}\left(\tilde{f}_{0}, \Lambda_{0}\right) & =-A_{1}\left(\tilde{f}_{0}, \Lambda_{0}\right) \frac{\partial}{\partial \tilde{f}_{0}}+\frac{1}{2} A_{2}\left(\tilde{f}_{0}, \Lambda_{0}\right) \frac{\partial^{2}}{\partial \tilde{f}_{0}^{2}},
\end{aligned}
$$

with the backward variables, $\tilde{f}_{0}$ and $\Lambda_{0}$. Here, $\mathcal{L}^{\dagger}$ is defined as the formal adjoint of the elliptical operator $\mathcal{L}$ (e.g., 

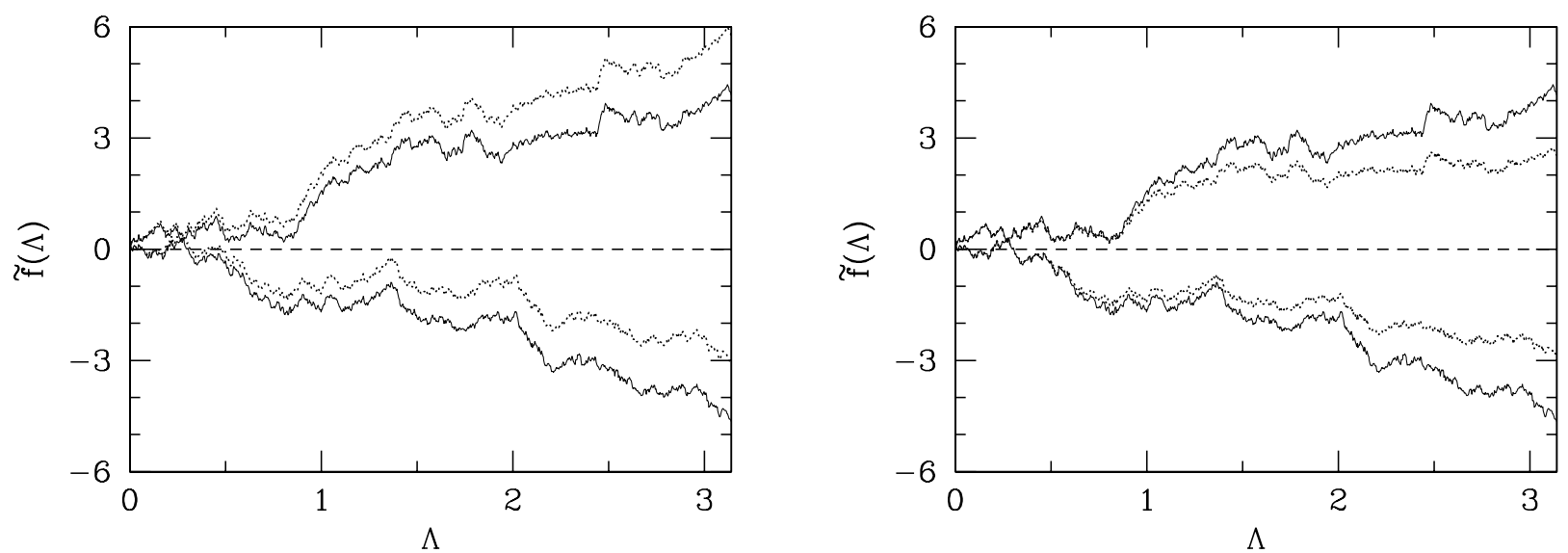

Fig. 1. Sketch of the large-scale behaviour of sample paths of low-order stochastic diffusion processes. Continuous lines: sample paths of a Wiener process with no drift term and the diffusion parameter $A_{2}=1$. Dotted lines: sample paths of diffusion processes with the same diffusion parameter but nonzero drift terms; left panel: order-0 process with $A_{1}=-0.5$ (Eq. (9)). Right panel: order-1 process with $A_{1}=0.2 \tilde{f}$

Kloeden \& Platen 1995, Sect. 2.4; Arnold 1974, Sect. 2.6). In Eq. (6) the backward variables $\tilde{f}_{0}$ and $\Lambda_{0}$, and in (7) the forward variables $\tilde{f}$ and $\Lambda$ are essentially constant and enter only through boundary conditions. The sign of $A_{1}$ is chosen to be consistent with (5). The Itô formula (see Eq. (A.2) in Appendix A) can be used to verify the equivalence of the Fokker-Planck equation and (5). For $\tilde{f}-\Lambda$ diffusion processes Bond et al. (1991) showed that Eq. (6) can be derived via the Chapman-Kolmogorov equation in the standard way. For these diffusion processes it is also straightforward to show the validity of Kolmogorov's backward equation. Hence, (5) can be used also for more general $\tilde{f}-\Lambda$ diffusion processes.

The Itô stochastic differential equation cannot be solved in general. However, as a guideline consider equilibrium solutions with $\partial \Pi / \partial \Lambda=0$. Simple solutions of (6) exist if $A_{2}$ is independent of $\tilde{f}$ and $\Lambda$, that is, $A_{2}=D=$ const. For this choice the correct form of $A_{1}$ is needed which is consistent with steady state. For $A_{1}$ independent of $\Lambda, A_{1}<0$ would lead to $\tilde{f}$ values "accelerated" to large $|\tilde{f}|$ with increasing $\Lambda$. Additional dependencies of $A_{1}$ on even powers of $\tilde{f}$ would not change the situation. However, if $A_{1}$ depends on odd powers of $\tilde{f}, A_{1}>0$ would lead for positive $\tilde{f}$ to be less positive and for negative $\tilde{f}$ to be less negative. Expanding $A_{1}$ in odd powers of $\tilde{f}$ thus yields stable deterministic states. In this respect important cases are the order- $\nu$ processes,

$$
\mathrm{d} \tilde{f}(\Lambda)=-A \tilde{f}^{\nu} \mathrm{d} \Lambda+\sqrt{D} \mathrm{~d} w(\Lambda), \quad(\nu=0 \text { or odd }) .(8)
$$

For $\nu \geq 3$, hitherto only small-noise expansions are known (e.g., Gardiner 1997, p.185). We are thus left with the Ansatz $\nu=0$ and $\nu=1$. Some historical notes on the resulting equations can be found in Wax (1954). Although it is not possible to give graphical presentations of sample paths of stochastic diffusion processes, Fig. 1 tries to compare trajectories of order- 0 and order- 1 diffusion processes obtained with

$$
\begin{aligned}
\tilde{f}\left(\Lambda_{i}\right)= & -A_{1}\left[\tilde{f}\left(\Lambda_{i-1}\right)\right] \Lambda_{i} \\
& +\sqrt{A_{2}\left[\tilde{f}\left(\Lambda_{i-1}\right)\right]} w_{i} \quad(i=1, \ldots, N),
\end{aligned}
$$

the $i$ th resolution variable, $\Lambda_{i}$, and $w_{i}$ from Wiener's construction as described in Itô \& McKean (1974),

$$
\begin{array}{r}
w_{i}=\frac{\Lambda_{i}}{\sqrt{\pi}} g_{0}+\sum_{n=1}^{m} \sum_{k=2^{n-1}}^{2^{n}-1} \sqrt{\frac{2}{\pi}} \frac{\sin \left(k \Lambda_{i}\right)}{k} g_{k} \\
\quad\left(0 \leq \Lambda_{i} \leq \pi\right),
\end{array}
$$

where $g_{k}, k \geq 0$ are independent and Gaussian distributed with zero mean and variance 1 . For $m \rightarrow \infty$, Eq. (10) converges uniformly in $0 \leq \Lambda_{i} \leq \pi$ with probability 1 and is a standard Brownian motion. The sample paths in Fig. 1 are computed with $m=10$ and $N=1000$ in the convergence range (larger $m$ values do not significantly improve the figure). For the order- 0 process we set $A_{1}\left[\tilde{f}\left(\Lambda_{i-1}\right)\right]=-\mu$, for the order- 1 process $A_{1}\left[\tilde{f}\left(\Lambda_{i-1}\right)\right]=A \tilde{f}\left(\Lambda_{i-1}\right)$, and $A_{2}\left[\tilde{f}\left(\Lambda_{i-1}\right)\right]=1$ for both (see Fig. 1 for more details). Figure 1 illustrates the exceedingly irregular motion of order- 0 processes with an almost infinite "speed", when $\tilde{f}$ and $\Lambda$ are regarded as the position and time of a Brownian particle, respectively. Order-0 processes have no stationary distributions, i.e., as $\Lambda \rightarrow \infty$, any finite point moves to infinity with probability 1 . Order- 1 processes include a linear drift term, which acts like dynamical friction, and which yields the only Gaussian Markov process in one real variable with a stationary distribution. The process is often used to model a more realistic Brownian motion, in which $\tilde{\Lambda}$ and $\tilde{\Lambda}^{\prime}$ are significantly correlated if $\left|\Lambda-\Lambda^{\prime}\right| \sim 1 / A$ (Eq. (A) in Appendix A).

In Sect. 4 it will be shown that each filter used to smooth Gaussian matter distributions and to extract masses of isolated objects is closely related to a specific diffusion process. To illustrate this point, sample paths 


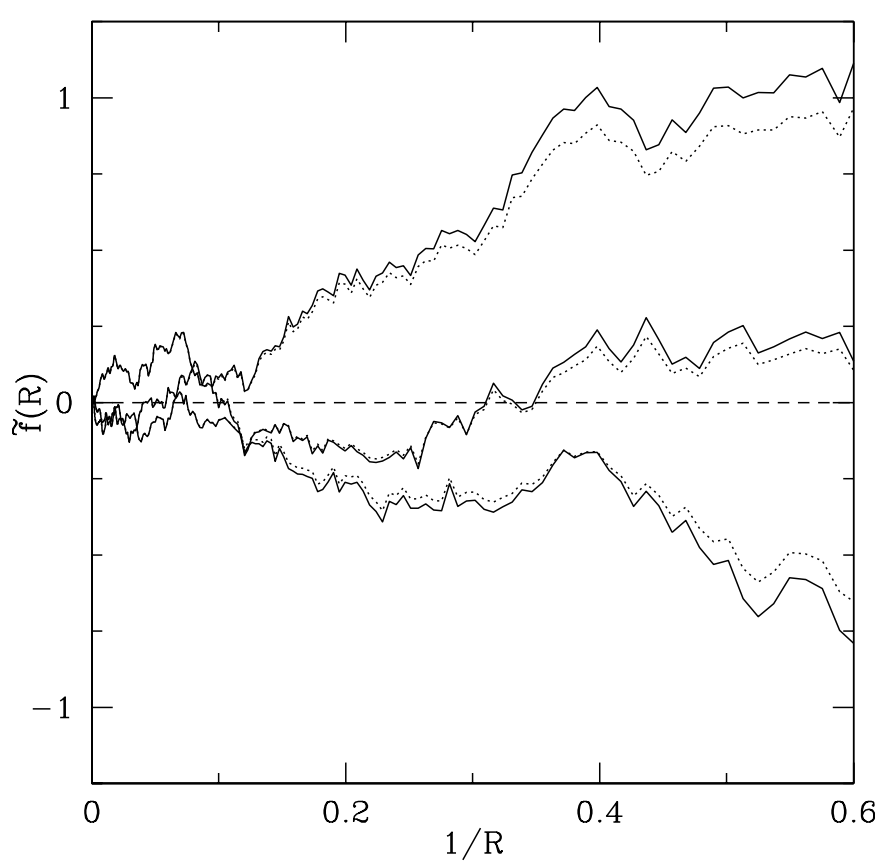

Fig. 2. Comparison of sample paths obtained for a high-density region (upper two trajectories), for a low-density region (lower two trajectories), and for an average-density region (middle two trajectories) by filtering a one-dimensional Gaussian random field (spectral index $n=0$ ) using a sharp $k$-space filter (continuous lines) and a truncated exponential filter (dotted lines) with $A=0.5$. The filter scale, $R$, is set to arbitrary physical units. The systematic differences between the trajectories obtained with the sharp $k$-space filter and with the truncated exponential filter reflect the differences between the trajectories of order- 0 and order- 1 diffusion processes shown in Fig. 1

are computed for specific locations (a high, average, lowdensity region) within a one-dimensional Gaussian random field which is smoothed with filter functions where the corresponding diffusion processes are known. Figure 2 shows this for the sharp $k$-space filter giving sample paths which correspond to order-0 diffusion processes (see Sect.6), and for the truncated exponential filter giving sample paths which correspond to order- 1 diffusion processes (see Sect.7). Despite the artificial smoothing of trajectories at large $1 / R$ caused by the limited numerical resolution in this scale range, the sample paths of the mathematical models of order- 0 and order- 1 diffusion processes shown in Fig. 1 and the sample paths obtained from filtered Gaussian random fields obtained with the process-specific filter functions shown in Fig. 2 look qualitatively quite similar. It is thus seen that order- 0 and order-1 diffusion processes are supposed to give a realistic description of the complex behaviour of the density contrasts at a given location in a Gaussian random field when filtered with process-specific filter functions and different filter widths.

Concerning the physical meaning of the diffusion and drift parameter it is important to realize that whereas the diffusion parameter basically determines the relation between immediately adjacing mass scales, the drift pa- rameter determines the relation between largely differing mass scales.

Ignoring for the moment the deterministic drift subprocesses, the drift-less diffusion process is assumed to be independent of mass and density contrast. The resulting random behaviour of the filtered density contrasts is thus determined by the inclusion or exclusion of a new independent $k$ shell. Because of the specific choice of the resolution variable (see Eq. (3)) and the normalization of the filter (see Sects. 6.2 and 7.2), the variance of the Gaussian noise process and thus of the value of the diffusion parameter, $A_{2}$, is per construction always unity. With the addition of a deterministic drift subprocess the idealistic pure diffusion process can be converted into a more realistic process because the idealized assumptions of the Wiener process can be relaxed.

The physical meaning of the drift parameter becomes clear from the deterministic motions induced (see Figs. 1 and 2). As an example, for $\Lambda$-independent drift parameters (the diffusion processes discussed here have this property) the potential

$U(\tilde{f})=\int^{\tilde{f}} A_{1}\left(\tilde{f}^{\prime}\right) \mathrm{d} \tilde{f}^{\prime}$

might be defined (e.g., Risken 1984, Chap. 5), leading for the order-1 process with $A>0$ to a parabolic potential which restricts the otherwise freely streaming trajectories to smaller values of $|\tilde{f}|$ (see right panel in Fig. 1). In Appendix A (Eq. (A.4) it is shown that the corresponding "external deterministic force" introduces realistic nonzero covariances between different $\tilde{f}(\Lambda)$, that is, it restricts the trajectories to a smaller $\tilde{f}$ interval around $\tilde{f}=0$ which has the following two related consequences: (1) It results in a weakening of the sharp $k$-space filter at larger comoving wavenumbers $k$ (see Sect.7.2). This is of great cosmological significance because the corresponding filters in configuration space are comparatively smooth (see Fig. 5) and thus frame a more realistic region of material that immersed into a particular halo. (2) It results in a correlation between different mass scales and thus effectively weakens the strict Markovian property, however, without leaving the general framework of Markovian processes. These correlations are expected to give more realistic formation histories and biasing schemes compared to the original PressSchechter approach.

On the other side, order-0 processes lead to systematic shifts of the trajectories in the direction of smaller or larger $\tilde{f}$ values (left panel in Fig. 1). This might be interpreted as a $\Lambda$ (mass)-dependent change of the critical density threshold, $f_{c}$, which will be discussed in more detail in Sect.6.1.

\section{Diffusion processes and mass functions}

In the following the general relation between diffusion processes and mass functions is developed. As already stated in Sect. 2 the excursion set formalism assumes that the cumulative mass fraction $n(>M) / n_{\text {tot }}$, where $n_{\text {tot }}$ is some 
normalization constant, is identified with the fraction of volume elements above $f_{\mathrm{c}}$ when smoothed with a filter of radius $\geq R(M)$ which is directly related to $\Lambda(R)$ (see below). At least for the high masses it seems reasonable to associate the change in total volume of the excursion region with the net mass in objects of this scale (see Bond et al. 1991, and the useful graphical visualisations given therein).

In the language of diffusion processes described in Sect. 3, the differential mass function is proportional to the probability that a particular trajectory is absorbed at the threshold $f_{\mathrm{c}}$ in a given $\Lambda$, i.e., mass range. It is thus necessary to investigate the first passage $\Lambda$ distribution of the trajectories supposing an absorbing barrier at $f_{\mathrm{c}}>\tilde{f}_{0}$. Let $\Pi\left(\tilde{f}_{0}, \tilde{f}, \Lambda\right)$ be the probability density to have trajectories at $\tilde{f}(\Lambda)$ and that no trajectory has reached $f_{\mathrm{c}}$ within the interval $(0, \Lambda)$. Notice the difference in the definition of $\Pi$ here and in Sect. 3 . Hence, the probability that absorption has not yet occured at $\Lambda$ is (sometimes the $\tilde{f}_{0}$ variable will be omitted in the argument lists of $F$, $\Pi$, and $g$ )

$F\left(\tilde{f}_{0}, f_{\mathrm{c}}, \Lambda\right)=\int_{-\infty}^{f_{\mathrm{c}}} \Pi\left(\tilde{f}_{0}, \tilde{f}, \Lambda\right) \mathrm{d} \tilde{f}$.

The lower limit, $\tilde{f}=-1$, must be extended formally to $-\infty$ in order to fulfill for all $\Lambda$ or mass scales under consideration the assumption of a Gaussian diffusion process. Notice that $F\left(\tilde{f}_{0}, \tilde{f}, \Lambda\right)$ and $\Pi\left(\tilde{f}_{0}, \tilde{f}, \Lambda\right)$ satisfy $(6,7)$. The probability density function of the first passage of trajectories at $\Lambda$ is determined by the "loss-rate" of sample paths at $\Lambda$, i.e.,

$g\left(\Lambda, f_{\mathrm{c}}, \tilde{f}_{0}\right)=-\frac{\partial}{\partial \Lambda} \int_{-\infty}^{f_{\mathrm{c}}} \Pi\left(\tilde{f}_{0}, \tilde{f}, \Lambda\right) \mathrm{d} \tilde{f}$.

In the following $g\left(\Lambda, f_{\mathrm{c}}, \tilde{f}_{0}\right)$ is called the pseudo mass function. The probability density function $g\left(\Lambda, f_{\mathrm{c}}, \tilde{f}_{0}\right)$ can be determined by solving Kolmogorov's forward Eq. (6) for $\Pi\left(\tilde{f}_{0}, \tilde{f}, \Lambda\right)$ under appropriate boundary conditions (mirror image method, see Sects.6.1 and 7.1). For $\Lambda$ independent $f_{\mathrm{c}}$, a second method determines the Laplace transform of $g\left(\Lambda, f_{\mathrm{c}}, \tilde{f}_{0}\right)$,

$g^{*}\left(\lambda, f_{\mathrm{c}}, \tilde{f}_{0}\right)=\int_{0}^{\infty} \mathrm{e}^{-\lambda \Lambda} g\left(\Lambda, f_{\mathrm{c}}, \tilde{f}_{0}\right) \mathrm{d} \Lambda$

and solves the Laplace-transformed Kolmogorov's backward Eq. (7) in conjunction with appropriate boundary conditions (Laplace transform method, see Sects. 6.1 and 7.1). The most difficult part of the latter method is the inversion of (14). To obtain the differential mass function,

$n(M)=\frac{\bar{\rho}_{0}}{M}\left|\frac{\mathrm{d} \Lambda(M)}{\mathrm{d} M}\right| g\left(\Lambda, f_{\mathrm{c}}, \tilde{f}_{0}\right)$,

we have to derive $\Lambda(M)$ which is closely related to the filter function of the diffusion process. For Gaussian matter distributions, this relation is obtained in the following way. In the first step, the equivalence between the mass variance (1) and the variance of the $\tilde{f}$ 's,

$$
\frac{1}{(2 \pi)^{3}} \int_{0}^{\infty} \mathrm{d}^{3} k\left|W_{R}(k)\right|^{2} P(k)=\operatorname{var}[\tilde{f}(\Lambda)],
$$

is used to derive the Fourier transform of the filter function, $W_{R}(k)$. The variances of the $\tilde{f}$ 's can be obtained with the Itô formalism in a straightforward way (see, e.g., Appendix A). In the second step, the resulting window function, $W_{R}(k)$, is transformed into configuration space to give $W_{R}(r)$, from which the filter volume is determined by $V(R)=1 / W_{R}(r=0)$. The mass associated with the filter is then obtained from $M(R)=\bar{\rho}_{0} V(R)$, where $\bar{\rho}_{0}$ is the present mean mass density of the Universe. Notice that this step follows the general conventions as introduced in Lacey \& Cole (1994). In the last step, the general relation between $\Lambda$ and $R$ (see Eq. (19)) is applied to give the wanted $\Lambda(M)$ relation. Section 5 presents a discussion of the general $\Lambda(R)$ relation.

\section{The $\Lambda-R$ resolution scale relation}

Within the framework of stochastic diffusion processes, mass functions are determined by the probabilities that the continuous sample paths of the diffusion process are first absorbed at $\tilde{f}=f_{\mathrm{c}}$. Analytical results are obtained by assuming no correlations between $\tilde{f}$ and its infinitesimal increments, d $\tilde{f}$, but (see, e.g., Eq. (A.8) for $A=0$ in Appendix A)

$$
\begin{aligned}
\mathrm{E}\left[\tilde{f}(R) \cdot \tilde{f}\left(R^{\prime}\right)\right] & =\frac{1}{(2 \pi)^{3}} \int \mathrm{d}^{3} k W_{R}(k) W_{R^{\prime}}^{*}(k) P(k) \\
& =\sigma^{2}\left(\max \left\{R, R^{\prime}\right\}\right) .
\end{aligned}
$$

The last equality can be achieved if the filter function, $W_{R}(k)$, has the form of the sharp $k$-space filter (Eq. (2)), and the resolution variable, $\Lambda$, is defined by $\Lambda=\sigma^{2}(R)$. This is the prescription of the fundamental stochastic diffusion process. Hence, the $\Lambda$-dependence of the probabilities $\Pi\left(\tilde{f}_{0}, \tilde{f}, \Lambda\right)$ can be obtained from the simplified Fokker-Planck equation,

$\frac{\partial}{\partial \Lambda} \Pi\left(\tilde{f}_{0}, \tilde{f}, \Lambda\right)=\frac{D}{2} \frac{\partial^{2}}{\partial \tilde{f}^{2}} \Pi\left(\tilde{f}_{0}, \tilde{f}, \Lambda\right)$,

with the diffusion coefficient, $D=1$. It is a classical exercise of the Itô stochastic formalism to show that (18) can be deduced from (5) and vice versa for the constants $A_{1}=0$ and $A_{2}=D$. Although these assumptions (including boundary conditions, for example, Eqs. (24)) suffice to derive the exact form and normalization of the PressSchechter function, they are for several reasons not very realistic. Zero-correlations between $\tilde{f}$ and its increments are only attainable if the stochastic force, $K$, has zero mean and the covariance $E\left[K\left(\Lambda^{\prime}\right) \cdot K(\Lambda)\right]=\operatorname{cov}\left(\Lambda^{\prime}-\Lambda\right)=$ $D \delta_{\mathrm{D}}\left(\Lambda^{\prime}-\Lambda\right)$. This gives a white noise power spectrum and thus an infinite variance. As $\Lambda \rightarrow 0$, solutions of the simplified Fokker-Planck equation tend to the Dirac delta distribution. As $\Lambda \rightarrow \infty$, the probabilities, $\Pi(\cdot)$, tend to 
zero, and no stationary solution exists. It is seen that in the same sense as the sharp $k$-space filter seems to be an idealistic choice to solve the mass function problem, the related fundamental stochastic diffusion process with its vanishing drift term must also be regarded as idealistic.

No consistent solutions are, however, attainable for $A_{1} \neq 0$ in combination with the original choice in the excursion set theory for the quasi time variable $\Lambda \equiv \sigma^{2}$, assuming that $\sigma^{2}$ is defined via a process-specific filter function and the power spectrum in the standard way. The inconsistency is seen by choosing, for example, $E\{\Delta \tilde{f} \mid \tilde{f}\}=A \tilde{f}$ in Eq. (3.4) of Bond et al. (1991), where $A$ is a constant independent of $\tilde{f}$ and $\Lambda$, that is, $A_{1}=A \tilde{f}$ in (5). For $A_{2}=D$, this is the order-1 or Ornstein-Uhlenbeck stochastic diffusion process. Appendix A summarizes some basic properties of this diffusion process. Assuming $A>0$, as $\Lambda \rightarrow \infty$ the variances (see Eq. (A.5)) tend to the finite value, $\sigma^{2}=D /(2 A)$, but in the excursion set theory it is assumed that $\sigma^{2}=\Lambda$ which tends to infinity. This contradiction shows that the specific choice, $\Lambda=\sigma^{2}$, is not suitable for a discussion of more general stochastic diffusion processes.

However, a general resolution scale relation, $\Lambda(R)$, is needed to handle different diffusion processes in an homogeneous way. The most natural choice is the $\Lambda(R)$ scale relation defined by the fundamental process,

$$
\begin{aligned}
\Lambda(R)=\frac{1}{2 \pi^{2}} \int_{0}^{\frac{1}{R}} \mathrm{~d} k k^{2} P(k) & =\frac{1}{2 \pi^{2}} \frac{P_{0}}{n+3} R^{-(n+3)} \\
\text { for } P(k) & =P_{0} k^{n} \quad(n>-3),
\end{aligned}
$$

as the common resolution scale for all diffusion processes investigated. A further motivation for this specific choice is given by the transformation property of the Wiener process. For example, the Wiener process, $\mathrm{d} \tilde{f}(\Lambda)=\mathrm{d} w(\Lambda)$, is converted into the Ornstein-Uhlenbeck process, $\mathrm{d} \tilde{f}(\Lambda)=-A \tilde{f} \mathrm{~d} \Lambda+\sqrt{D} \mathrm{~d} w(\Lambda)$, by the transformation $\tilde{f}(\Lambda)=h(\Lambda) \tilde{f}_{\mathrm{w}}[T(\Lambda)]$, with the amplitude transformation $h(\Lambda)=\mathrm{e}^{-A \Lambda}$, and the resolution variable transformation $T(\Lambda)=D /(2 A) \mathrm{e}^{2 A \Lambda}$, where again - and this is the important point - the resolution variable of the fundamental process, $\Lambda$, sets the basic resolution scale. Notice that the instrumental distinction between $\Lambda$ and $\sigma^{2}$ is necessary because $\Lambda \neq \sigma^{2}$ in general. Only for the fundamental (Wiener) process we have $\Lambda=\sigma^{2}$. In the following sections pseudo mass functions, $g\left(\Lambda, f_{\mathrm{c}}, \tilde{f}_{0}\right)$, are derived and transformed into "true" differential mass functions, $n(M)$, using the Eqs. (15), (16).

\section{Mass functions from order-0 (Wiener) stochastic diffusion processes}

Order-0 Gaussian stochastic diffusion processes are characterized by the constants $A_{1}=-\mu$ (or $A=-\mu$ and $\nu=0$ in Eq. (8)), and $A_{2}=D$ in (5), used to write the Itô stochastic differential equation in the form

$\mathrm{d} \tilde{f}(\Lambda)=\mu \mathrm{d} \Lambda+\sqrt{D} \mathrm{~d} w(\Lambda)$, with the solution

$\tilde{f}(\Lambda)=\tilde{f}_{0}+\mu \Lambda+\sqrt{D} \int_{0}^{\Lambda} \mathrm{d} w\left(\Lambda^{\prime}\right)$,

and hence the instantaneous mean $E[\tilde{f}(\Lambda)]=\tilde{f}_{0}+\mu \Lambda$, and the instantaneous variance $\operatorname{var}[\tilde{f}(\Lambda)]=D \Lambda$. The corresponding Kolmogorov's forward equation is

$\frac{D}{2} \frac{\partial^{2} \Pi}{\partial \tilde{f}^{2}}-\mu \frac{\partial \Pi}{\partial \tilde{f}}=\frac{\partial \Pi}{\partial \Lambda} \quad\left(-\infty<\tilde{f}<f_{\mathrm{c}}\right)$.

The filtered density contrasts are restricted to values below the zero redshift critical overdensity, e.g., $f_{\mathrm{c}}=$ $(3 / 20)(12 \pi)^{(2 / 3)} \Omega_{0}^{0.0185}$ for the spherical symmetric cosmological infall model (Gunn \& Gott 1972; Bertschinger 1985; Navarro et al. 1996). Notice that in (22), $\Pi$ is the probability density for $\tilde{f}$ conditional to $\tilde{f}(0)=\tilde{f}_{0}$, and that the process sample paths do not reach $f_{\mathrm{c}}$ in the resolution interval $(0, \Lambda)$. The cumulative distribution function, $\bar{F}\left(\tilde{f}_{0}, f_{\mathrm{c}}, \Lambda\right)=1-F\left(\tilde{f}_{0}, f_{\mathrm{c}}, \Lambda\right)$, defined by the probability density of first absorbed sample paths, is directly related to the pseudo mass function via Eqs. (12), (13), and (15). We thus have

$\bar{F}\left(\tilde{f}_{0}, f_{\mathrm{c}}, \Lambda\right)=1-\int_{-\infty}^{f_{\mathrm{c}}} \Pi\left(\tilde{f}_{0}, \tilde{f}, \Lambda\right) \mathrm{d} \tilde{f}$,

and compute $\bar{F}\left(\tilde{f}_{0}, f_{\mathrm{c}}, \Lambda\right)$ solving (22) under the boundary conditions

$\Pi(\tilde{f}, \Lambda=0)=\delta_{\mathrm{D}}(\tilde{f}), \quad \Pi\left(\tilde{f}=f_{\mathrm{c}}, \Lambda\right)=0 \quad(\Lambda>0)$.

Whereas the first equation reflects the deterministic initial state of the diffusion process, the second equation gives the appropriate boundary condition for an absorbing barrier at $f_{\text {c }}$ (e.g., Cox \& Miller 1965, p. 219): assume a nonvanishing infinitesimal variance when $\tilde{f}=f_{\mathrm{c}}$. The probability that the trajectory is absorbed within $\Delta \Lambda$ can be approximated by the product of the probability that the trajectory is near (i.e., slightly below) $f_{\mathrm{c}}$, and the probability that the next increment $\Delta \tilde{f}-$ which is proportional to $\sqrt{\Delta \Lambda}$ (see Sect. 3 ) - carries it beyond $f_{\mathrm{c}}$. However, the latter probability is assumed to be always larger zero. In order to keep the probability density (i.e., the absorption probability per $\Delta \Lambda$ ) finite that the trajectory is absorbed when $\tilde{f} \rightarrow f_{\mathrm{c}}$, the former probability must be zero because $\sqrt{\Delta \Lambda} / \Delta \Lambda \rightarrow \infty$ when $\Delta \Lambda \rightarrow 0$.

\subsection{Pseudo mass functions}

The unconstrained case of Eq. (22) has the general solution (Fig. 3)

$$
\Pi_{1}\left(\tilde{f}_{0}, \tilde{f}, \Lambda\right)=\frac{1}{\sqrt{2 \pi D \Lambda}} \exp \left[-\frac{\left(\tilde{f}-\tilde{f}_{0}-\mu \Lambda\right)^{2}}{2 D \Lambda}\right],
$$

where the sample paths start at $\tilde{f}_{0}$. Exploiting the symmetry of the diffusion process, which still holds for $\mu \neq 0$, a solution is obtained by placing one source of sample 

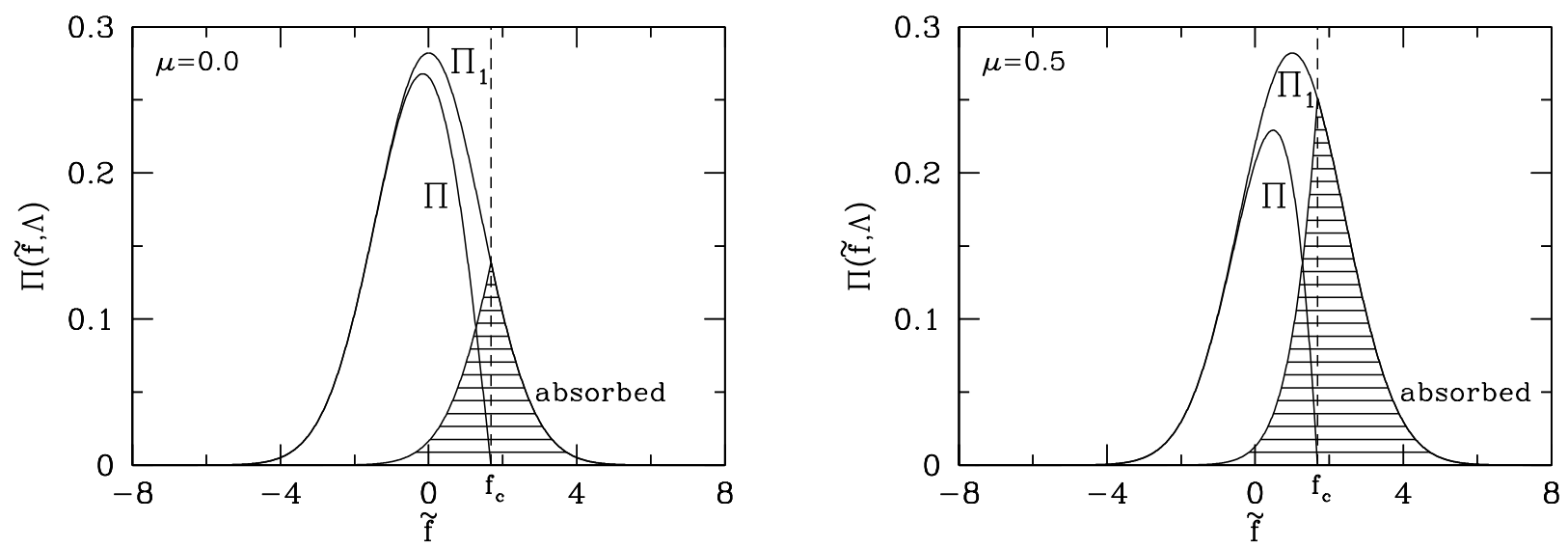

Fig. 3. Probability density distributions for unconstrained sample paths $\left(\Pi_{1}\right.$, see Eq. (25)), for nonabsorbed sample paths $(\Pi$, see Eq. (26)), and for absorbed sample paths (hatched areas) for order-0 processes without drift $(\mu=0$, left panel) and with the drift parameter $\mu=0.5$ (right panel)

paths at $\tilde{f}_{0}=0$, and a second source at $\tilde{f}_{0}=2 f_{\tilde{c}}(\operatorname{mir}-$ ror image method), that is, $\Pi(\tilde{f}, \Lambda)=\Pi_{1}(0, \tilde{f}, \Lambda)+$ $\alpha \Pi_{1}\left(2 f_{\mathrm{c}}, \tilde{f}, \Lambda\right)$, where the factor $\alpha$ is determined by the condition $\Pi\left(f_{\mathrm{c}}, \Lambda\right)=0$. The solution gives the probability of nonabsorbed sample paths and takes the form

$$
\begin{aligned}
\Pi(\tilde{f}, \Lambda)= & \frac{1}{\sqrt{2 \pi D \Lambda}}\left\{\exp \left[-\frac{(\tilde{f}-\mu \Lambda)^{2}}{2 D \Lambda}\right]-\exp \left(\frac{2 \mu f_{\mathrm{c}}}{D}\right)\right. \\
& \left.\times \exp \left[-\frac{\left(\tilde{f}-2 f_{\mathrm{c}}-\mu \Lambda\right)^{2}}{2 D \Lambda}\right]\right\}\left(-\infty<\tilde{f}<f_{\mathrm{c}}\right) .
\end{aligned}
$$

It is seen that the second source in (26), i.e., the last term on the right-hand side, has a negative amplitude and acts as a "sink" for sample paths. The drift parameter, $\mu$, introduces a mass $(\Lambda)$-dependent shift of $\Pi_{1}$ which increases $(\mu>0)$ or decreases $(\mu<0)$ the probability for absorbed sample paths (see shaded regions in Fig. 3). Although the threshold, $f_{\mathrm{c}}$, is assumed to be mass-independent, the systematic drift might be interpreted as an effective change of the critical threshold in a mass-dependent way. Notice that a possible physical interpretation is that for high mass (low- $\Lambda$ ) clumps the effect of the surrounding volume elements is small so that the collaps will be spherical along all three directions with $f_{\mathrm{c}}$ close to the spherical collaps model (Bernadeau 1994). For small mass (high $\Lambda$ ) clumps the effects of the surrounding volume elements might be more significant and can thus alter the effective value of $f_{\mathrm{c}}$.

Substituting (26) into (13) gives the pseudo mass function for the order-0 diffusion process,

$$
\begin{aligned}
g\left(\Lambda, f_{\mathrm{c}}\right) & =\frac{\bar{F}\left(f_{\mathrm{c}}, \Lambda\right)}{\mathrm{d} \Lambda} \\
& =\frac{f_{\mathrm{c}}}{\sqrt{2 \pi D}}\left(\frac{1}{\Lambda}\right)^{\frac{3}{2}} \exp \left[-\frac{\left(f_{\mathrm{c}}-\mu \Lambda\right)^{2}}{2 D \Lambda}\right] .
\end{aligned}
$$

The mirror image method is widely used in solving problems of heat conduction and heat diffusion (e.g., Landau \& Lifschitz 1966). It "reduces" the solution of the diffusion problem originally restricted to a specific domain of diffusion space to an infinite region.
Since the absorbing barrier is $\Lambda$-independent, exact pseudo mass functions can also be deduced by taking Laplace transforms in the Kolmogorov's backward equation (Laplace transform method) giving the partial differential equation for $g^{*}\left(\lambda, f_{\mathrm{c}}, \tilde{f}_{0}\right)$,

$$
\begin{aligned}
\frac{D}{2} \frac{\partial^{2}}{\partial \tilde{f}_{0}^{2}} g^{*}\left(\lambda, f_{\mathrm{c}}, \tilde{f}_{0}\right)+\mu \frac{\partial}{\partial \tilde{f}_{0}} g^{*}\left(\lambda, f_{\mathrm{c}}, \tilde{f}_{0}\right) & \\
& =\lambda g^{*}\left(\lambda, f_{\mathrm{c}}, \tilde{f}_{0}\right) .
\end{aligned}
$$

The backward equation is the appropriate equation because of the fixed final state $f_{\mathrm{c}}$, we seek for solutions $g^{*}$ as a function of the backward variable $\tilde{f}_{0}$ and set $\tilde{f}_{0}=0$ later. For order-0 diffusion processes the general solution of $(28)$ is

$g^{*}\left(\lambda, f_{\mathrm{c}}, \tilde{f}_{0}\right)=A \mathrm{e}^{\tilde{f}_{0} H_{1}(s)}+B \mathrm{e}^{\tilde{f}_{0} H_{2}(s)}$.

Inserting (29) in (28) gives a quadratic equation for the $H$ 's, and thus, after applying the condition $g^{*}\left(\lambda, f_{\mathrm{c}}, \tilde{f}_{0}\right) \leq$ 1 and $g^{*}\left(\lambda, f_{\mathrm{c}}, \tilde{f}_{0}=f_{\mathrm{c}}\right)=1$ (immediate absorption) from which the constants $A$ and $B$ are determined, the Laplace transform of the pseudo mass function (for $\tilde{f}_{0}=0$ ),

$g^{*}\left(\lambda, f_{\mathrm{c}}\right)=\exp \left[\frac{f_{\mathrm{c}}}{D}\left(\mu-\sqrt{\mu^{2}+2 D \lambda}\right)\right]$.

Inversion of (30) again gives (27): both the mirror image and the Laplace transform method lead to the same result. For $\mu \rightarrow 0$, Eq. (27) is the well-known Press-Schechter pseudo mass function with the correct normalization (see Fig. 4).

\subsection{Mass functions}

Equating the process variance, $D \Lambda$, of the $\tilde{f}$ 's with the mass variance, $\sigma^{2}(R)$, and using (19) gives the relation which determines the filter function for this process,

$$
\begin{aligned}
\frac{1}{2 \pi^{2}} \int_{0}^{\infty} \mathrm{d} k k^{2}\left|W_{R}(k)\right|^{2} P(k) & =D \Lambda \\
& =\frac{D}{2 \pi^{2}} \int_{0}^{\frac{1}{R}} \mathrm{~d} k k^{2} P(k) .
\end{aligned}
$$



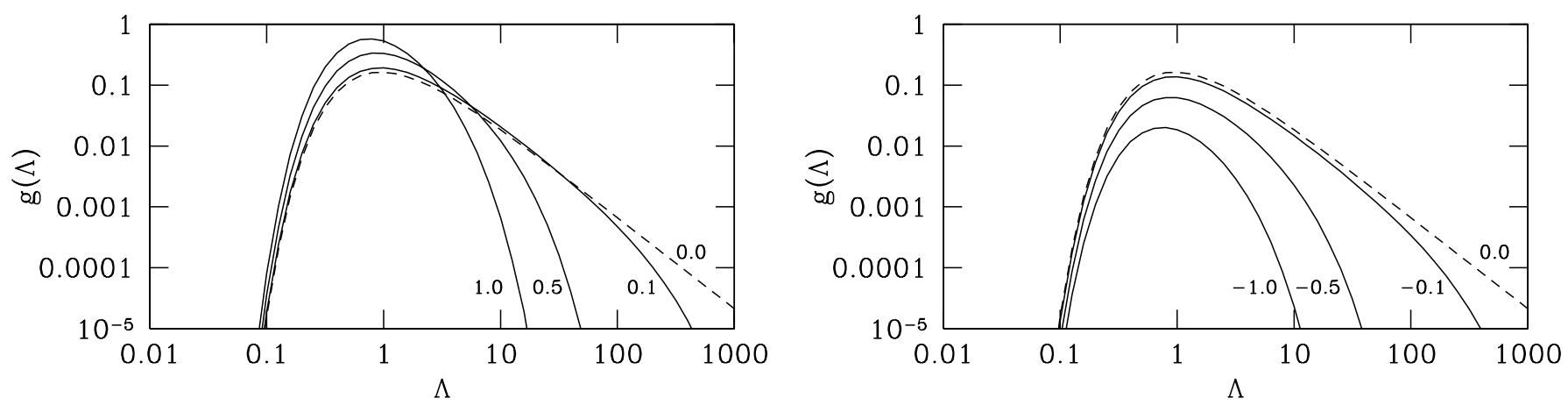

Fig. 4. Pseudo mass functions from order-0 processes for different $\mu$ values (continuous lines). For comparison, the PressSchechter pseudo mass function $(\mu=0)$ is given (dashed lines). Both the mirror image and the Laplace transform method give the same results
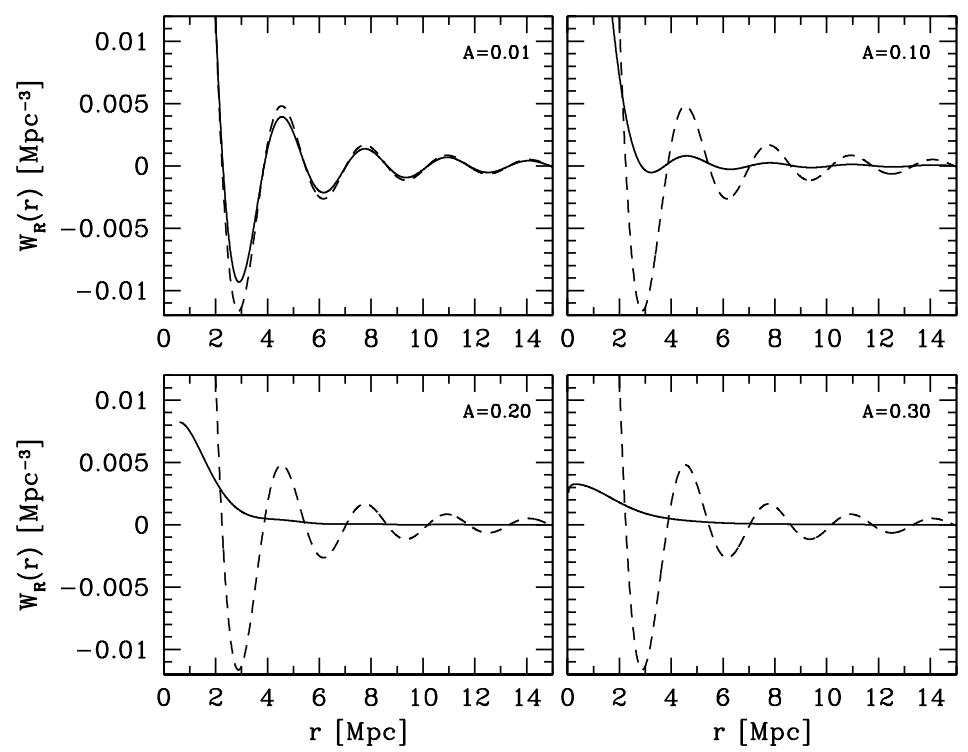

Fig. 5. Filter functions in configuration space: the sharp $k$-space filter (dashed lines), and the truncated exponential filter (continuous lines) for the filter scale, $R=0.25 \mathrm{Mpc}$. The drift parameters have values between zero (dashed line) and 0.3 . The amplitude of the power spectrum is $P_{0}=200 \mathrm{Mpc}$, and the spectral index $n=-2$ (normalization and shape similar to a CDM power spectrum). The normalized Hubble constant, $h=0.5$, is used in units of $H_{0}=100 \mathrm{~km} \mathrm{~s}^{-1} \mathrm{Mpc}^{-1}$

As expected, the filter function is the sharp $k$-space filter (Eq. (2)). The normalization of the filter function yields $D=1$. Note that the relation between $\Lambda$ and $\sigma^{2}$ is simply given by $\sigma^{2}(\Lambda)=\Lambda$. In configuration space, the volume and the mass within the filter radius $R$ as well as the sharp $k$-space filter function (Fig. 5) are

$$
\begin{aligned}
& V_{\mathrm{S}}=\frac{1}{W_{R}(r=0)}=6 \pi^{2} R^{3}, \quad M_{\mathrm{S}}=6 \pi^{2} \bar{\rho}_{0} R^{3}, \\
& W_{R}(r)=\frac{1}{2 \pi^{2} r^{3}}\left[\sin \left(\frac{r}{R}\right)-\left(\frac{r}{R}\right) \cos \left(\frac{r}{R}\right)\right] .
\end{aligned}
$$

For a scale-invariant power spectrum, $P(k)=P_{0} k^{n}$, the $M(\Lambda)$ relation becomes

$$
M_{\mathrm{S}}=6 \pi^{2} \bar{\rho}_{0}\left[\frac{P_{0}}{2 \pi^{2}(n+3) \Lambda}\right]^{\frac{3}{n+3}} \text {. }
$$

The condition for hierarchical growth of structure (variance decreases with mass) is $n>-3$. Notice that $\Lambda$ is basically determined by the filter scale $R$, and by the power spectrum of the mass density distribution. This has important implications when mass functions, $n(M)$, are derived by using Eq. (15). The $\Lambda(M)$ relation (we omit the subscript of $M)$,

$\Lambda(M)=\frac{P_{0}}{2 \pi^{2}(n+3)}\left(\frac{6 \pi^{2} \bar{\rho}_{0}}{M}\right)^{\frac{n+3}{3}}$,

where $\bar{\rho}_{0}=2.775510^{11} \Omega_{0} h^{2} M_{\odot} \mathrm{Mpc}^{-3}$, gives the mass function,

$$
\begin{aligned}
n(M)= & \frac{\left(2 \pi^{2}\right)^{\frac{3}{n+3}}}{18 \pi^{2} \sqrt{2 \pi}}(n+3)^{\frac{6+n}{3+n}} \frac{f_{\mathrm{c}}}{M P_{0}^{\frac{3}{n+3}}} \Lambda^{\frac{3-n}{6+2 n}} \\
& \times \exp \left[-\frac{\left(f_{\mathrm{c}}-\mu \Lambda\right)^{2}}{2 \Lambda}\right] .
\end{aligned}
$$

Ratios of the mass functions induced by order- 0 processes with positive drift parameters to the Press-Schechter mass function are shown in Fig. 6. Equation (36) differs from the standard Press-Schechter function by the extra $\mu \Lambda$ term in 

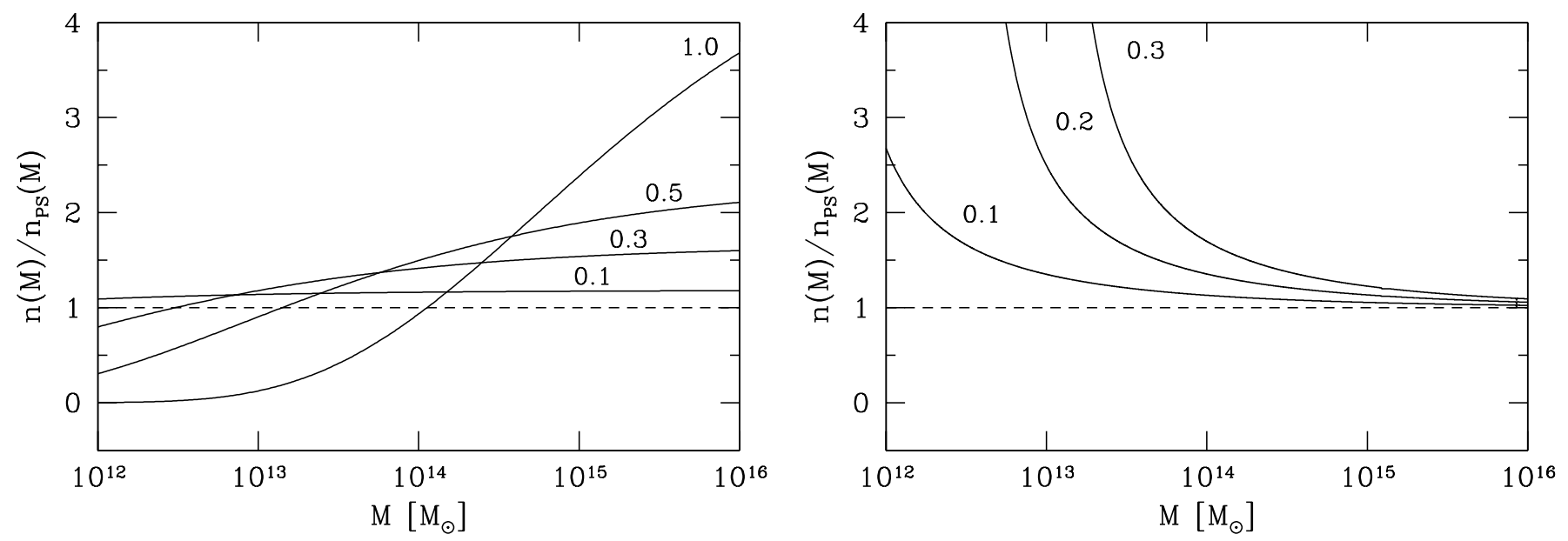

Fig. 6. Ratios of the mass functions induced by order-0 (left) and order-1 processes (right). A scale-invariant power spectrum with the index $n=-2$ and the amplitude $P_{0}=200 \mathrm{Mpc}$, the critical density contrast, $f_{\mathrm{c}}=1.68$, the normalized Hubble constant, $h=0.5$, and the density parameter, $\Omega_{0}=1.0$, are used. The different curves are labeled by the value of the corresponding drift parameter. Masses are given in solar units

the exponential. As expected positive drift parameters increase the fraction of high $\tilde{f}$ and hence the number of highmass objects to the expense of objects with lower mass. For $\mu \rightarrow 0$, Eq. (36) converges to the Press-Schechter mass function.

\section{Mass functions from order-1 (Ornstein-Uhlenbeck) stochastic diffusion processes}

Order-1 Gaussian stochastic diffusion processes are characterized by the parameters $A_{1}=A \tilde{f}$ (or $\nu=1$ in Eq. (8)), and $A_{2}=D$, used to write the Itô stochastic differential equation in the form

$\mathrm{d} \tilde{f}(\Lambda)=-A \tilde{f} \mathrm{~d} \Lambda+\sqrt{D} \mathrm{~d} w(\Lambda)$.

Historical notes on this equation can be found in Wax (1954). Some basic informations about this process are given in Appendix A. The formal solution for $\tilde{f}_{0}=0$ is

$\tilde{f}(\Lambda)=\sqrt{D} \int_{0}^{\Lambda} \mathrm{e}^{-A\left(\Lambda-\Lambda^{\prime}\right)} \mathrm{d} w\left(\Lambda^{\prime}\right)$,

yielding the instantaneous mean, $E[\tilde{f}(\Lambda)]=0$, and the instantaneous variance,

$\operatorname{var}[\tilde{f}(\lambda)]=\frac{D}{2 A}\left(1-\mathrm{e}^{-2 A \Lambda}\right)$.

It is straightforward to write down the Kolmogorov's forward and backward Eqs. (6), (7) for this process. The boundary conditions are identical to those already used for the order- 0 processes in Sect. 6 .

\subsection{Pseudo mass functions}

Since the absorbing barrier at $f_{\mathrm{c}}$ is constant, the Laplace transform method (Sect. 6.1) can be applied. The Laplace transform of the corresponding Kolmogorov's backward equation (e.g., Beekman 1975 and the references given therein) leads to the pseudo mass function (definitions of the specific mathematical functions used in the following can be found in, e.g., Abramowitz \& Stegun 1979),

$$
\begin{aligned}
g^{*}\left(\lambda, f_{\mathrm{c}}\right) & =\exp \left(-\frac{A S^{2}}{2 D}\right) \frac{D_{-\lambda / A}(0)}{D_{-\lambda / A}\left(-f_{\mathrm{c}} \sqrt{\frac{2 A}{D}}\right)} \\
& =\frac{\Phi_{D, A}(\lambda, 0)}{\Phi_{D, A}\left(\lambda, f_{\mathrm{c}}\right)}
\end{aligned}
$$

where $D_{\alpha}(z)$ is the parabolic cylinder function with $\alpha=$ $-\Lambda / A$,

$$
\begin{aligned}
\Phi_{D, A}\left(\lambda, f_{\mathrm{c}}\right)= & \frac{1}{\Gamma\left(\frac{\lambda}{2 A}\right) \Gamma\left(\frac{1+\lambda / A}{2}\right)} \\
& \times \sum_{n=0}^{\infty} \frac{\left(2 f_{\mathrm{c}} \sqrt{\frac{A}{D}}\right)^{n}}{n !} \Gamma\left(\frac{n+\lambda / A}{2}\right)
\end{aligned}
$$

an auxillary function, and $\Gamma(\cdot)$ the Euler gamma function. The inversion of the Laplace transform appeared recently in the mathematical literature (see Ricciardi \& Sato 1988) and involves the zeros $\lambda=\lambda_{\pi}\left(f_{\mathrm{c}}\right)$ of $(41)$ - they are all negative - giving the pseudo mass function

$g\left(\Lambda, f_{\mathrm{c}}\right)=\sum_{p=0}^{\infty} K_{p}\left(f_{\mathrm{c}}\right) \exp \left[\lambda_{p}\left(f_{\mathrm{c}}\right) \Lambda\right]$,

where

$$
\begin{aligned}
K_{p}^{-1}\left(f_{\mathrm{c}}\right) & =\frac{1}{2 A} \sum_{n=0}^{\infty} \frac{\left(2 f_{\mathrm{c}} \sqrt{\frac{A}{D}}\right)^{n}}{n !} \frac{\Gamma\left(\frac{n+\lambda_{p} / A}{2}\right)}{\Gamma\left(\frac{\lambda_{p}}{2 A}\right)} \\
\times & {\left[\Psi\left(\frac{n+\lambda_{p} / A}{2}\right)-\Psi\left(\frac{1+\lambda_{p} / A}{2}\right)-\Psi\left(\frac{\lambda_{p}}{2 A}\right)\right], }
\end{aligned}
$$



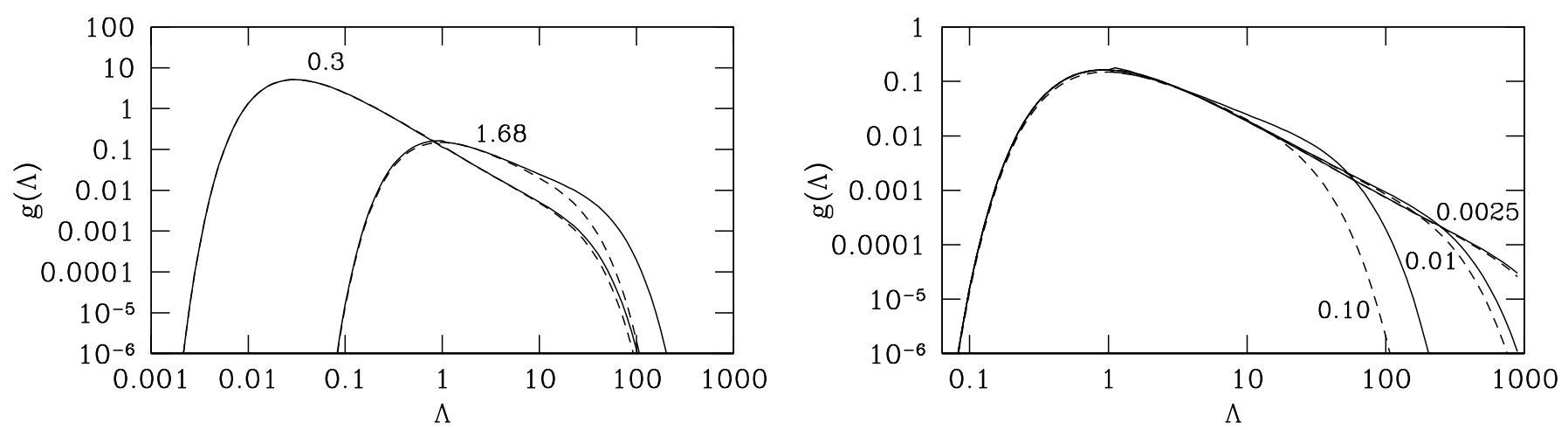

Fig. 7. Pseudo mass functions for order-1 processes obtained with the Laplace transform method (continuous lines) and with the mirror image method (dashed lines). Left $(A=0.1): f_{\mathrm{c}}=0.3$ and 1.68. Right $\left(f_{\mathrm{c}}=1.68\right): A=0.10,0.01,0.0025$. Note the different scales of the two figures

and $\Psi(\cdot)$ being the digamma function. The asymptotic behaviour of (42) may be summarized as

$g\left(\Lambda, f_{\mathrm{c}}\right) \sim\left\{\begin{array}{rc}\frac{f_{\mathrm{c}}}{\sqrt{2 \pi D}}\left(\frac{1}{\Lambda}\right)^{\frac{3}{2}} \mathrm{e}^{-f_{\mathrm{c}}^{2} /(2 \Lambda)}: & \Lambda \text { small } \\ K_{0}\left(f_{\mathrm{c}}\right) \mathrm{e}^{-\left|\lambda_{0}\right| \Lambda}: & \Lambda \text { large }\end{array}\right.$.

The pseudo mass function can thus be expressed as the sum of exponential functions with negative exponents $\left(\lambda_{p}<0\right)$ and reduces to single exponentials for small and for large $\Lambda$. For $A \rightarrow 0$, Eq. (42) tends to the PressSchechter pseudo mass function.

These results are compared to the closed-form analytic approximation obtained with the mirror image method (see Sect. 6.1). The unconstrained Fokker-Planck equation has the solution

$$
\begin{aligned}
\Pi_{1}\left(\tilde{f}_{0}, \tilde{f}, \Lambda\right)= & \frac{1}{\sqrt{2 \pi}}\left[\frac{2 A}{D\left(1-\mathrm{e}^{-2 A \Lambda}\right)}\right]^{\frac{1}{2}} \\
& \times \exp \left[-\frac{A\left(\tilde{f}-\tilde{f}_{0} \mathrm{e}^{-A \Lambda}\right)^{2}}{D\left(1-\mathrm{e}^{-2 A \Lambda}\right)}\right],
\end{aligned}
$$

where all sample paths start at $\tilde{f}_{0}$. The superposition of two sources of sample paths gives the constrained solution

$$
\begin{aligned}
& \Pi(\tilde{f}, \Lambda)=\frac{1}{\sqrt{2 \pi}}\left[\frac{2 A}{D\left(1-\mathrm{e}^{-2 A \Lambda}\right)}\right]^{\frac{1}{2}} \\
& \times\left[\mathrm{e}^{-\frac{A \tilde{f}^{2}}{D\left(1-\mathrm{e}^{-2 A \Lambda}\right)}}-\mathrm{e}^{\frac{4 f_{\mathrm{c}}^{2} A \mathrm{e}^{-A \Lambda}\left(\mathrm{e}^{-A \Lambda}-1\right)}{D\left(1-\mathrm{e}^{-2 A \Lambda}\right)}} \mathrm{e}^{-\frac{A\left(\tilde{f}-2 f_{\mathrm{c}} \mathrm{e}^{-A \Lambda}\right)^{2}}{D\left(1-\mathrm{e}^{-2 A \Lambda}\right)}}\right] .
\end{aligned}
$$

Taking into account the boundary conditions (24) we obtain

$$
\begin{aligned}
g\left(\Lambda, f_{\mathrm{c}}\right)= & \frac{f_{\mathrm{c}}}{\sqrt{2 \pi D}}\left(\frac{2 A}{1-\mathrm{e}^{-2 A \Lambda}}\right)^{\frac{3}{2}} \\
& \times \exp \left[-\frac{A f_{\mathrm{c}}^{2}}{D\left(1-\mathrm{e}^{-2 A \Lambda}\right)}-A \Lambda\right] .
\end{aligned}
$$

As for order-0 processes, $\Lambda$ is given by (19). Note that for fixed $f_{\mathrm{c}}$ all pseudo mass functions agree in the low$\Lambda$ range, i.e., for large masses; differences are seen in the high- $\Lambda$ range, i.e., for low masses. For small $f_{\mathrm{c}}$ or small $A$ the solutions obtained with the Laplace transform method can be approximated remarkebly well by the closed-form analytic results obtained with the mirror image method although the strict symmetry of the diffusion process is distorted by the $\tilde{f}$-dependence of the drift coefficient. For small $A$ both solutions tend to the Press-Schechter pseudo mass function (see Figs. 7 and 8).

\subsection{Mass functions}

Equating the process variance (39) with the mass variance, $\sigma^{2}(R)$, and using (19) gives the relation which determines the window function,

$$
\begin{aligned}
\frac{1}{2 \pi^{2}} \int_{0}^{\infty} \mathrm{d} k k^{2} & \left|W_{R}(k)\right|^{2} P(k)=\frac{D}{2 A} \\
& \times\left\{1-\exp \left[-\frac{A}{\pi^{2}} \int_{0}^{\frac{1}{R}} \mathrm{~d} k k^{2} P(k)\right]\right\} .
\end{aligned}
$$

For a given diffusion process the filter function and thus the region where material ultimately collapses to form a virialized halo cannot be chosen in any case independently from the power spectrum of the Gaussian random field.

For scale-invariant power spectra the filter function consistent with the density field and diffusion process is

$W_{R}(k)=\left\{\begin{array}{cl}\exp \left(-\frac{A P_{0} k^{n+3}}{2 \pi^{2}(n+3)}\right): & k \leq 1 / R \\ 0: & \text { else }\end{array}\right.$

This is a particular solution of the singular integral Eq. (48) found "by eye". Possible ambiguous solutions in the form of strongly fluctuating window functions in $k$ space are physically not very interesting and can thus be disgarded. The normalization, $D=1$, ensures $W_{R}(k=$ $0)=1$. For $A>0$ it is an exponential filter truncated at the wavenumber $1 / R$. For large $R$ Eq. (49) resembles the sharp $k$-space filter whereas for small $R$ the wings of the exponential get more important. Consider first a power spectrum with $n=-2$ (the more general case $n>-3$ is treated thereafter). For this realistic case the truncated exponential filter (49) transformed into configuration 
space is

$$
\begin{aligned}
W_{R}(r)= & \frac{\mathrm{e}^{-\frac{A P_{0}}{2 \pi^{2} R}}}{2 \pi^{2} r\left[\left(\frac{A P_{0}}{2 \pi^{2}}\right)^{2}+r^{2}\right]}\left\{-\left[\frac{A P_{0}}{2 \pi^{2} R}+\frac{\left(\frac{A P_{0}}{2 \pi^{2}}\right)^{2}-r^{2}}{\left(\frac{A P_{0}}{2 \pi^{2}}\right)^{2}+r^{2}}\right]\right. \\
& \times \sin \left(\frac{r}{R}\right)-\left[\frac{r}{R}+\frac{A P_{0} r}{\pi^{2}\left(\left(\frac{A P_{0}}{2 \pi^{2}}\right)^{2}+r^{2}\right)}\right] \\
& \left.\times \cos \left(\frac{r}{R}\right)+\frac{A P_{0} r \mathrm{e}^{\frac{A P_{0}}{2 \pi^{2} R}}}{\pi^{2}\left[\left(\frac{A P_{0}}{2 \pi^{2}}\right)^{2}+r^{2}\right]}\right\}
\end{aligned}
$$

Equation (50) corresponds to (33) as $A \rightarrow 0$ (Fig. 5). The cumulative mass and volume at the filter scale $R$ are determined by the inverse of $W_{R}(r)$ as $r \rightarrow 0$, resulting in

$$
\begin{aligned}
M_{\mathrm{E}} & =\bar{\rho}_{0} V_{\mathrm{E}}, \\
V_{\mathrm{E}} & =2 \pi^{2}\left(\frac{A P_{0}}{2 \pi^{2}}\right)^{2} \mathrm{e}^{\frac{A P_{0}}{2 \pi^{2} R}} \\
& \times\left[\frac{4 \pi^{2}}{A P_{0}}\left(\mathrm{e}^{\frac{A P_{0}}{2 \pi^{2} R}}-1\right)-\frac{2}{R}-\frac{A P_{0}}{2 \pi^{2} R^{2}}\right]^{-1},
\end{aligned}
$$

with the limits

$$
V_{\mathrm{E}}=\left\{\begin{array}{rl}
6 \pi^{2} R^{3}: & R \gg \frac{A P_{0}}{2 \pi^{2}} \\
\frac{\pi^{2}}{\left(2 \pi^{2}\right)^{3}}\left(A P_{0}\right)^{3}: & R \ll \frac{A P_{0}}{2 \pi^{2}}
\end{array} .\right.
$$

The $M(\Lambda)$ relation thus becomes

$$
\begin{aligned}
M_{\mathrm{E}}= & \bar{\rho}_{0}\left(\frac{A P_{0}}{2 \pi^{2}}\right)^{2} P_{0} \mathrm{e}^{A \Lambda} \\
& \times\left[\frac{2}{A}\left(\mathrm{e}^{A \Lambda}-1\right)-2 \Lambda-A \Lambda^{2}\right]^{-1},
\end{aligned}
$$

which can be solved numerically for $\Lambda$ to compute the mass function (Eq. (15)). Ratios of the mass functions induced by order- 1 processes with positive drift parameters to the Press-Schechter mass function are shown in Fig. 6. Equation (53) can be approximated by

$M_{\mathrm{E}} \approx \frac{3 \bar{\rho}_{0} P_{0}^{3}}{4 \pi^{4}}\left(\frac{1}{\Lambda^{3}}+\frac{A^{3}}{6}\right)$,

leading to the $\Lambda(M)$ relation (we omit the subscript of $M$ )

$\Lambda \approx\left(\frac{4 \pi^{4} M}{3 \bar{\rho}_{0} P_{0}^{3}}-\frac{A^{3}}{6}\right)^{-\frac{1}{3}}$.

and with the approximation (47) to the mass function

$$
\begin{aligned}
n(M) \approx & \frac{4 \pi^{4}}{9 \sqrt{2 \pi}} \frac{f_{\mathrm{c}}}{M P_{0}^{3}} \Lambda^{4}\left(\frac{2 A}{1-\mathrm{e}^{-2 A \Lambda}}\right)^{\frac{3}{2}} \\
& \times \exp \left(-\frac{A f_{\mathrm{c}}^{2}}{1-\mathrm{e}^{-2 A \Lambda}}-A \Lambda\right) .
\end{aligned}
$$

It is seen that the mass function gives more objects with low mass and similar numbers of objects with high mass compared to the Press-Schechter case (see Fig.6). The differences increase with increasing drift parameter and illustrate the effects of the different shapes of the filter functions (see Fig. 5) on the mass function.

For scale-invariant power spectra with index $n>-3$, expansion of (49) and pice-wise integration gives the general $M(\Lambda)$ relation,

$$
\begin{aligned}
& M_{\mathrm{E}}(\Lambda)=\bar{\rho}_{0} V_{\mathrm{E}} \\
& V_{\mathrm{E}}^{-1}=W_{R}(r=0) \\
&=\frac{1}{2 \pi^{2}} \int_{0}^{\infty} \mathrm{d} k k^{2} W_{R}(k)=\frac{\sum_{m=0}^{\infty} \frac{(-1)^{m} A^{m}}{m !\left[m\left(\frac{n+3}{3}\right)+1\right]} \Lambda^{m}}{6 \pi^{2}\left[\frac{P_{0} \Lambda}{2 \pi^{2}(n+3)}\right]^{\frac{3}{n+3}}}
\end{aligned}
$$

from which the mass function can be obtained numerically in the same way as from (53). For $A \rightarrow 0$ only the $m=0$ term in the numerator of (57) gives a contribution to the sum, thus reproducing the volume of the sharp $k$ space filter.

\section{Summary and discussion}

The present paper concentrates on the development of methods to derive analytic mass functions for given stochastic diffusion processes. For practical purposes the analysis is restricted to order- 0 and order- 1 processes. Whereas order-0 processes are characterized by drift coefficients which are independent of the filtered density contrast, order-1 processes have a linear dependence on the filtered density contrast. It is seen that in order- 1 stochastic diffusion processes, although derived within the framework of Markovian processes, realistic nonzero covariances appear in a very natural way without sampling over the diffusion (merging) history. The correlation between two mass scales gets important especially for small masses. For realistic cases the filter functions corresponding to the diffusion processes are derived. Whereas for all order-0 processes the filter function is the sharp $k$-space filter, for order-1 processes exponential filters are found. The effects of non-sharp $k$-space filters on the mass function are in the low-mass range where an excess compared to the standard Press-Schechter expectation is found. Future investigations should work with higher order diffusion processes in order to get more realistic potential wells (determined by the drift term) for sample diffusion. Of special interest are asymmetric potentials which might give a more appropriate handling of the asymmetric distribution of $\tilde{f}$ within $[-1, \infty)$.

Nonetheless, do we really need a discussion of more general diffusion processes? From the practical point of view one might argue that the Press-Schechter mass function, although derived under very specific assumptions, is well confirmed by comparison with $N$-body simulations (e.g., Efstathiou et al. 1988; White et al. 1993; Lacey \& Cole 1994; Mo et al. 1996) and should thus suffice to be used as a simple fitting formula. The mass resolution and dynamic range of the simulations are, however, too low to test the prescription in detail. New results show indeed significant deviations from the analytical expectation (e.g., Jing 1998; Sheth \& Tormen 1999; 

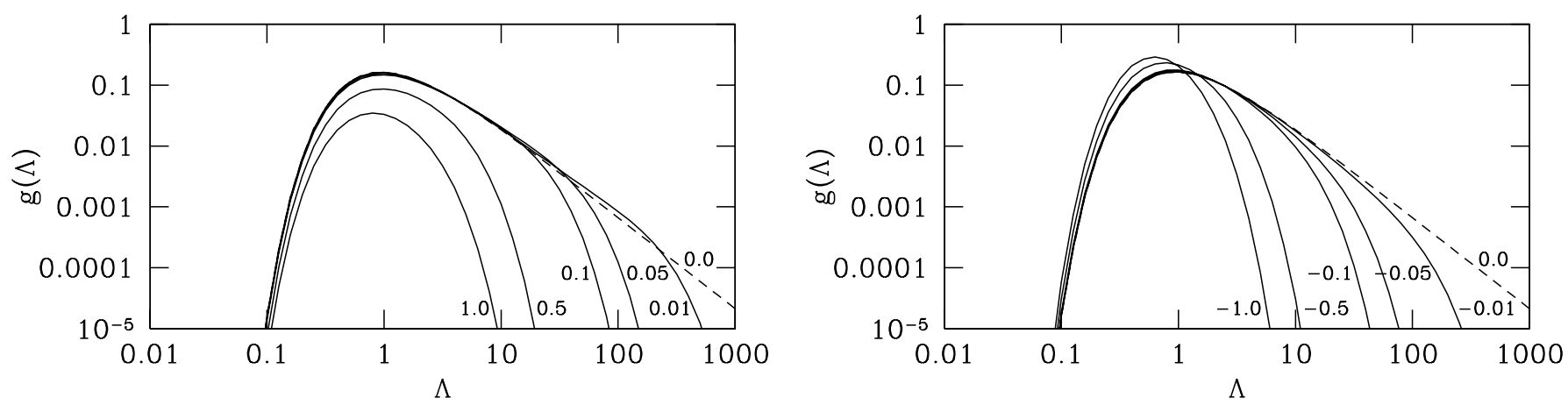

Fig. 8. Pseudo mass functions from order-1 processes obtained with the mirror image method for different $A$ values (continuous lines). For comparison, the Press-Schechter pseudo mass function $(A=0)$ is given (dashed lines)

Governato et al. 1999). In the light of the discussion given above one might expect such deviations from the PressSchechter formula. Consequently one should avoid any unnecessary conditions on the type of diffusion process. The new coefficients offer a more flexible summary of the new mass functions.

In forthcoming papers we want to derive consistent mass and filter functions for more complicated stochastic diffusion processes and compare the results with detailed $N$-body simulations and observed mass and luminosity functions derived from physically well-defined X-ray selected samples of clusters of galaxies.

Acknowledgements. We would like to thank Jörg Retzlaff for a critical reading of the manuscript and the anonymous referee for many useful comments. P. S. thanks for support by the Verbundforschung under the grant No. 50 OR 970835.

\section{Appendix A: Order-1 stochastic diffusion processes}

The Itô stochastic differential equation of these processes is

$\mathrm{d} \tilde{f}(\Lambda)=-A \tilde{f} \mathrm{~d} \Lambda+\sqrt{D} \mathrm{~d} w(\Lambda)$.

Early versions of this equation can be found in Uhlenbeck \& Ornstein (1930) and Doob (1942). Equation (A.1) can be solved by introducing the variable $\tilde{u}=\tilde{\mathrm{f}} \mathrm{e}^{A \Lambda}$, and using the Itô formula for a sufficiently smooth function, $f$, of $\tilde{f}(\Lambda)$ satisfying $(5)$,

$$
\begin{aligned}
\mathrm{d} f[\tilde{f}(\Lambda)]= & {\left[\frac{\partial f}{\partial \Lambda}-A_{1}(\tilde{f}(\Lambda), \Lambda) \frac{\partial f}{\partial \tilde{f}}+\frac{1}{2} A_{2}(\tilde{f}(\Lambda), \Lambda) \frac{\partial^{2} f}{\partial \tilde{f}^{2}}\right] } \\
& \times \mathrm{d} \Lambda+\sqrt{A_{2}(\tilde{f}(\Lambda), \Lambda)} \frac{\partial f}{\partial \tilde{f}} \mathrm{~d} w(\Lambda),
\end{aligned}
$$

where the partial derivatives are evaluated at $(\Lambda, \tilde{f}(\Lambda))$. For $f$ linear in $\tilde{f}$ we have $\partial^{2} f / \partial \tilde{f}^{2}=0$, and (A.2) reduces to the usual chain rule. If $f=\tilde{u}$ this gives $\mathrm{d} \tilde{u}=$ $\sqrt{D} \mathrm{e}^{A \Lambda} \mathrm{d} w(\Lambda)$, which can be integrated and yields for the initial condition, $\tilde{f}(0)=0$,

$\tilde{f}(\Lambda)=\sqrt{D} \int_{0}^{\Lambda} \mathrm{e}^{-A\left(\Lambda-\Lambda^{\prime}\right)} \mathrm{d} w\left(\Lambda^{\prime}\right)$.
The increments, $\mathrm{d} w(\Lambda)$, of the fundamental (Wiener) process have a Gaussian distribution (Sect. 3). Hence, the linear superposition in (A.3) shows that the $\tilde{f}$ are again Gaussian distributed with zero mean, the covariance

$E\left[\tilde{f}\left(\Lambda_{1}\right) \tilde{f}\left(\Lambda_{2}\right)\right]=$

$D \cdot E\left[\int_{0}^{\Lambda_{1}} \mathrm{e}^{-A\left(\Lambda_{1}-\Lambda_{1}^{\prime}\right)} \mathrm{d} w\left(\Lambda_{1}^{\prime}\right) \int_{0}^{\Lambda_{2}} \mathrm{e}^{-A\left(\Lambda_{2}-\Lambda_{2}^{\prime}\right)} \mathrm{d} w\left(\Lambda_{2}^{\prime}\right)\right]$

$$
=\frac{D}{2 A}\left[\mathrm{e}^{-A\left|\Lambda_{1}-\Lambda_{2}\right|}-\mathrm{e}^{-A\left(\Lambda_{1}+\Lambda_{2}\right)}\right],
$$

and the instantaneous variance

$\operatorname{var}[\tilde{f}(\Lambda)]=\frac{D}{2 A}\left(1-\mathrm{e}^{-2 A \Lambda}\right)$.

Nonzero covariances between $\tilde{f}(\Lambda)$ and the increments $\Delta \tilde{f}(\Lambda+f \Lambda)$ lead to more realistic correlations between different parts of each sample path, and thus to more realistic shapes of the corresponding process filters. For $A>0$, and $\Lambda_{1}, \Lambda_{2} \gg 1$, the last term in (A) can be neglected, and the process has, as expected, a stationary covariance function which decreases exponentially with increasing distance $\left|\Lambda_{1}-\Lambda_{2}\right|$, corresponding to a non-white power spectrum. For $A<0$ less fast decreasing covariances are found.

The process can be generalized to the case with $\Lambda$ dependent coefficients,

$\mathrm{d} \tilde{f}(\Lambda)=-A(\Lambda) \tilde{f} \mathrm{~d} \Lambda+\sqrt{D(\Lambda)} \mathrm{d} w(\Lambda)$.

For $\tilde{f}(\Lambda=0)=0$ the solution of (A.6) is

$\tilde{f}(\Lambda)=\int_{0}^{\Lambda} \mathrm{d} w\left(\Lambda^{\prime}\right) \sqrt{D\left(\Lambda^{\prime}\right)} \exp \left[-\int_{\Lambda^{\prime}}^{\Lambda} A(s) \mathrm{d} s\right]$,

giving the covariance

$$
\begin{aligned}
& E\left[\tilde{f}\left(\Lambda_{1}\right) \tilde{f}\left(\Lambda_{2}\right)\right]=\int_{0}^{\min \left(\Lambda_{1}, \Lambda_{2}\right)} \mathrm{d} \Lambda^{\prime} D\left(\Lambda^{\prime}\right) \\
& \times \exp \left[-\int_{\Lambda^{\prime}}^{\Lambda_{1}} A(s) \mathrm{d} s-\int_{\Lambda^{\prime}}^{\Lambda_{2}} A\left(s^{\prime}\right) \mathrm{d} s^{\prime}\right],
\end{aligned}
$$

and the instantaneous variance

$\operatorname{var}[\tilde{f}(\Lambda)]=\int_{0}^{\Lambda} \mathrm{d} \Lambda^{\prime} D\left(\Lambda^{\prime}\right) \exp \left[-\int_{\Lambda^{\prime}}^{\Lambda} 2 A(s) \mathrm{d} s\right]$. 


\section{References}

Abramowitz, M., \& Stegun, I. A. 1979, Handbook of mathematical functions (Dover, New York)

Arnold, L. 1974, Stochastic Differential Equations: Theory and Applications (John Wiley and Sons, New York)

Beekman, J. A. 1975, J. App. Prob., 12, 107

Bernardeau, F. 1994, ApJ, 427, 51

Bertschinger, E. 1985, ApJS, 58, 39

Binggeli, B., Sandage, A. R., \& Tammann, G. A. 1988, ARA\&A, 26, 509

Böhringer, H., et al. 1998, The Messenger, 94, 21

Bond, J. R., Cole, S., Efstathiou, G., \& Kaiser, N. 1991, ApJ, 379,440

Borgani, S., Rosati, P., Tozzi, P., \& Norman, C. 1999, ApJ, 517,40

Bower, R. J. 1991, MNRAS, 248, 332

Cavaliere, A., \& Menci, N. 1994, ApJ, 435, 528

Chiu, W. A., Ostriker, J. P., \& Strauss, M. 1998, ApJ, 494, 479

Cole, S., \& Kaiser, N. 1988, MNRAS, 233, 637

Cole, S., \& Kaiser, N. 1989, MNRAS, 237, 1127

Cox, D. R., \& Miller, H. D. 1965, The Theory of Stochastic Processes (Chapmann Hall, London)

Doob, J. L. 1942, Ann. Math., 43, 351

Efstathiou, G., Frenk, C. S., White, S. D. M., \& Davis, M. 1988, MNRAS, 235, 715

Eke, V. R., Cole, S., \& Frenk, C. S. 1996, MNRAS, 282, 263

Ellis, R. S., Colless, M., Broadhurst, T., Heyl, J., \& Glazebrook, K. 1996, MNRAS, 280, 235

Friedman, A. 1975, Stochastic Differential Equations and Applications, vol. 1 (Academic Press, New York)

Gardiner, C. W. 1997, Handbook of Stochastic Methods (Springer Verlag, Berlin)

Governato, F., Babul, A., Quinn, T., et al. 1999, MNRAS, 307, 949

Gunn, J. E., \& Gott, J. R. III. 1972, ApJ, 176, 1

Guzzo, L., et al. 1999, The Messenger, 95, 27

Henry, J. P., \& Arnaud, K. A. 1991, ApJ, 372, 410

Itô, K., \& McKean Jr., H. P. 1974, Diffusion Processes and their Sample Paths (Springer-Verlag, Berlin), 21

Jedamzik, K. 1995, ApJ, 448, 1

Jing, Y. P. 1998, ApJ, 503, L9

Kauffmann, G., \& White, S. D. M. 1993, MNRAS, 261, 921

Kitayama, T., \& Suto, Y. 1996, ApJ, 469, 480
Kloeden, P. E., \& Platen, E. 1995, Numerical Solution of Stochastic Differential Equations (Springer Verlag, Berlin)

Lacey, C., \& Cole, S. 1993, MNRAS, 262, 627

Lacey, C., \& Cole, S. 1994, MNRAS, 271, 676

Landau, L. D., \& Lifschitz, E. M. 1966, Hydrodynamik (Akademie Verlag, Berlin)

Lee, J., \& Shandarin, S. F. 1998, ApJ, 500, 14

Lilje, P. B. 1992, ApJ, 386, L33

Lilly, S. J., Le Fevre, O., Crampton, D., Hammer, F., \& Tresse, L. 1995, ApJ, 455, 50

Lucchin, F., \& Matarrese, S. 1988, ApJ, 330, 535

Matarrese, S., Coles, P., Lucchin, F., \& Moscardini, L. 1997, MNRAS, 286, 115

Mathiesen, B., \& Evrard, A. E. 1998, MNRAS, 295, 769

Mo, H. J., \& White, S. D. M. 1996, MNRAS, 282, 347

Mo, H. J., Jing, Y. P., \& White, S. D. M. 1996, MNRAS, 282 1096

Monaco, P. 1995, ApJ, 447, 23

Narayan, R., \& White, S. D. M. 1988, MNRAS, 231, 97p

Navarro, J. F., Frenk, C. S., \& White, S. D. M. 1996, ApJ, 462, 563

Peebles, P. J. E. 1990, ApJ, 365, 27

Peacock, J. A. 1999, Cosmological Physics (Cambridge Univ. Press, Cambridge)

Peacock, J. A., \& Heavens, A. F. 1990, MNRAS, 243, 133

Press, W. H., \& Schechter, P. 1974, ApJ, 187, 425

Ricciardi, L. M., \& Sato, S. 1988, J. App. Prob., 25, 43

Risken, H. 1984, The Fokker-Planck Equation (SpringerVerlag, Berlin)

Sheth, R. K., \& Tormen, G. 1999, MNRAS, 308, 119

Uhlenbeck, G. E., \& Ornstein, L. S. 1930, Phys. Rev., 36, 823

Valageas, P., \& Schaeffer, R. 1997, A\&A, 328, 435

Wax, N. 1954, Selected papers on noise and stochastic processes (Dover Publ. Inc., New York)

White, S. D. M. 1996, in Cosmology and Large-Scale Structure, ed. R. Schaeffer, J. Silk, M. Spiro, \& J. Zinn-Justin (Elsevier, Dordrecht), 349

White, S. D. M. 1997, in The Evolution of the Universe, ed. G. Börner, \& S. Gottlöber (Wiley Sons, Chicester), 227

White, S. D. M., \& Frenk, C. S. 1991, ApJ, 379, 25

White, S. D. M., Efstathiou, G., \& Frenk, C. S. 1993, MNRAS, 262, 1023

Wiener, N. 1930, Acta Math., 55, 117

Yano, T., Nagashima, M., \& Gouda, N. 1996, ApJ, 466, 1 